\title{
Spectral measurement of the Hall angle response in normal state cuprate superconductors
}

\author{
M. Grayson ${ }^{1,2, *}$, L. B. Rigal ${ }^{1, \dagger}$, D. C. Schmadel ${ }^{1}$, H. D. Drew ${ }^{1,3}$, P.-J. Kung ${ }^{4}$ \\ ${ }^{1}$ Department of Physics, University of Maryland, College Park, MD 20742 \\ ${ }^{2}$ Walter Schottky Institut, Technische Universität München, D-85748 Garching, Germany \\ ${ }^{3}$ Center for Superconductivity Research, University of Maryland, College Park, MD 20742 \\ ${ }^{4}$ Advanced Fuel Research, Inc., East Hartford, CT
}

(29 May 2002)

We measure the temperature and frequency dependence of the complex Hall angle for normal state $\mathrm{YBa}_{2} \mathrm{Cu}_{3} \mathrm{O}_{7}$ films from dc to far-infrared frequencies $\left(20-250 \mathrm{~cm}^{-1}\right)$ using a new modulated polarization technique. We determine that the functional dependence of the Hall angle on scattering does not fit the expected Lorentzian response. We find spectral evidence supporting models of the Hall effect where the scattering $\Gamma_{H}$ is linear in $\mathrm{T}$, suggesting that a single relaxation rate, linear in temperature, governs transport in the cuprates.

The normal state Hall effect in cuprate superconductors exhibits an anomalous temperature dependence that cannot be explained using conventional transport theory for metals. According to the simple Drude model, the resistivity of a metal and the cotangent of its Hall angle $\cot \left(\theta_{H}\right)=\frac{\sigma_{x x}}{\sigma_{x y}}$, should share the same temperature dependence, both proportional to the scattering rate of the charge carriers. However, the normal state resistance of cuprate superconductors is linear with temperature, $\rho \sim T$, while the Hall angle has a robust $\cot \left(\theta_{H}\right) \sim T^{2}$ behavior [1] over a wide range of oxygen doping [2], and with substitutional doping [3] in a variety of the cuprates [4. This apparent duality of scattering rates characterizes the anomalous Hall transport in the cuprates. Several theories approached the problem assuming that two scattering rates were in fact involved, beginning with the spin-charge separation model of Anderson wherein the two species of quasiparticles each relaxed at the different observed rates. [5] Subsequent explanations focused either on alternative non-Fermi liquid mechanisms 6. 7] or on the effects of $k$-space scattering anisotropies 8 11. The common feature of all the above theories is a dominant term that is linear in the scattering rate, $\cot \left(\theta_{H}\right) \sim \gamma_{H}$. In contrast, a recent theory by Varma and Abrahams 12 treats anisotropic scattering in a marginal Fermi-liquid and predicts a square-scattering response, $\cot \left(\theta_{H}\right) \sim \gamma_{H}^{2}$.

These different models can be distinguished at finite frequency. The linear- and square-scattering models correspond to Lorentzian and square-Lorentzian spectral responses respectively, and although Hall experiments have been performed at finite frequencies [13 15], this paper is the first to study both temperature and frequency dependence of the Hall response in a frequency range that discerns a lineshape and extrapolates to the dc limit.

We begin by reviewing the concept of a frequency dependent Hall angle [16] using the Drude model as an example of a Lorentzian response. All parameters are implicitly spectral, i.e. $\theta_{H}=\theta_{H}(\omega)$, and in the present case of strong scattering, $\tan (\theta) \simeq \theta<<1$. Quasiparticles circling at the cyclotron frequency $\omega_{H}^{*}=e B / m c$ traverse a fraction $\omega_{H}^{*} \tau_{H}^{*}$ of a cyclotron orbit during the time $\tau_{H}^{*}$ between scattering events. The dc longitudinal current $j_{x}$ is thereby deflected into $j_{y}$ by this small arc angle $\theta_{H} \simeq \frac{j_{y}}{j_{x}}=\omega_{H}^{*} \tau_{H}^{*}=\omega_{H}^{*} / \gamma_{H}^{*}$, where $\gamma_{H}^{*}$ is defined as the Hall scattering rate. For the ac response, we substitute $\gamma_{H}^{*} \rightarrow \gamma_{H}^{*}-i \omega$ yielding a Lorentzian:

$$
\theta_{H}(\omega)=\frac{\omega_{H}^{*}}{\gamma_{H}^{*}-i \omega}
$$

Linear scattering models of the anomalous Hall transport predict this same Lorentzian form for the frequency response near the dc limit.

For the experiment at infrared frequencies, the Hall angle $\theta_{H}$ cannot be measured directly but must be deduced from transmission studies of polarized light $13-15$. The measurable quantity, the Faraday angle $\theta_{F}$, is the angle of rotation of polarized light induced after passing through a thin conducting film in the presence of a normal magnetic field $B$. In the thin film limit $(d<<\lambda, \delta)$ with $d$ the film thickness, $\lambda$ the wavelength, and $\delta$ the penetration depth, $\theta_{H}$ follows from $\theta_{F}$ according to Maxwell's equations:

$$
\theta_{H}=\left(\frac{1+n}{Z_{o} \sigma_{x x}}+1\right) \theta_{F}
$$

$\sigma_{x x}$ is the experimentally determined complex sheet conductivity, $Z_{o}$ the free space impedance, and $n=3.4$ the refractive index of the Si substrate. With our highly conductive films the term in parenthesis is near unity, so the functional dependence on $\sigma_{x x}$ is minimal. $\theta_{F}$ and $\theta_{H}$ are both causal response functions, so their Re and Im parts obey Kramers-Kronig relations and correspond to real space rotation and ellipticity, respectively [16].

The cuprate sample investigated was a pulsed-laser deposited, twinned film of $500 \AA \mathrm{YBa}_{2} \mathrm{Cu}_{3} \mathrm{O}_{7}$ on a $1 \times 1 \mathrm{~cm}^{2}$ $\times 350 \mu \mathrm{m}$ insulating silicon substrate, with an intermediate strain relieving layer of $100 \AA$ yttrium stabilized 
zirconate [13]. Partial data on a second sample confirmed the results reported here. The sample was mounted in an $8 \mathrm{~T}$ Oxford Spectromag cryostat with the $B$-field oriented along the optical axis, normal to the sample surface. Two $8 \mathrm{~cm}$ diameter Kapton cryostat windows on either side of the sample allowed direct optical access. Sourced by a broadband quartz $\mathrm{Hg}$ arc lamp, the spectra were measured using a step-scan Fourier transform interferometer with wire-grid polarized beam-splitters having a density of 40 wires per $\mathrm{mm}$. The novel technique consisted of a mechanically rotating optical element which modulated the polarization of light incident on the sample, and the transmitted signal was measured with a bolometer detector using standard lockin techniques at harmonics of the rotator frequency. Measurements of cyclotron resonance in GaAs verified the experimental technique.

Schematics for measuring $\operatorname{Re}\left(\theta_{F}\right)$ and $\operatorname{Im}\left(\theta_{F}\right)$ are shown in the insets of Fig. 11. To measure the Faraday angle $\operatorname{Re}\left(\theta_{F}\right)$, polarized light was projected through a mechanically rotating linear polarizer with rotation angle $\phi(t)=2 \pi f_{\text {rot }} t,\left(f_{\text {rot }}=70 \mathrm{~Hz}\right)$. The light then passed through the sample, striking a stationary polarizer before reaching the bolometer detector. The phase shift in the second harmonic of the bolometer signal is identically the Faraday rotation, $\operatorname{Re}\left(\theta_{F}\right)=\operatorname{Re}\left(t_{x y} / t_{x x}\right)$, where $t_{i j}$ is the transmittance tensor relating the transmitted field in the $j$ direction to the incident field in the $i$ direction 15 .

$\operatorname{Im}\left(\theta_{F}\right)$ is measured analogously (inset Fig. 1), with the light modulated into right and left elliptical polarizations by a mechanically rotating wave plate and by omitting the polarizer previously in front of the detector. The waveplate shifts the optical phase of the field component along its extraordinary axis by the retardance $\beta(\omega)$. In this case the in-phase second harmonic response is

$$
P=2\left|E_{i}\right|^{2} \sin (\beta) \operatorname{Im}\left(t_{x x}^{*} t_{x y}\right)
$$

Calibrating separately $\beta(\omega)$ and the transmitted spectral intensity, $S \simeq \frac{\left|E_{i}\right|^{2}}{2}\left(t_{x x}^{*} t_{x x}\right)$, we find,

$$
\operatorname{Im}\left(\theta_{F}\right)=\operatorname{Im}\left(\frac{t_{x y}}{t_{x x}}\right)=\left[\frac{P}{4 S \times \sin (\beta)}\right]
$$

Fig. 11 plots the directly measured $\operatorname{Re}\left(\theta_{F}\right)$ and $\operatorname{Im}\left(\theta_{F}\right)$ parts of the Faraday angle. The agreement of $\operatorname{Im}\left(\theta_{F}\right)$ with the Kramers-Kronig (K-K) transform of $\operatorname{Re}\left(\theta_{F}\right)$ confirms the consistency of our measurements, and justifies the use of the lower noise, wider bandwidth K-K transform to represent $\operatorname{Im}\left(\theta_{F}\right)$ in the following calculations.

Combining this complex $\theta_{F}$ with the complex longitudinal conductivity determined from extended-Drude fits to the transmission data 17,18] (inset, Fig. 2), the Hall angle is determined over the full $20-250 \mathrm{~cm}^{-1}$ range using Eq. 2 (Fig. 2). At low frequencies, we see that $\operatorname{Re}\left(\theta_{H}^{-1}\right)$ extrapolates to the dc transport values measured on a separate sample using the Van der Pauw geometry, and the dc points exhibit the $T^{2}$ law universally observed in the cuprates.
We first check consistency with the linear-scattering (Lorentzian) form of Refs. [5 11] by solving Eq. 11 for the Lorentzian parameters $\omega_{H}^{*}(\omega)=-\omega / \operatorname{Im}\left[\theta_{H}^{-1}(\omega)\right]$ and $\gamma_{H}^{*}(\omega)=\omega_{H}(\omega) \operatorname{Re}\left[\theta_{H}^{-1}(\omega)\right]$ in Fig. 3. This parametrization demonstrates that $\theta_{H}$ is not Lorenztian over the full frequency range since $\gamma_{H}(\omega)$ shows additional frequency dependence, decreasing significantly above $100 \mathrm{~cm}^{-1}$ particularly at low temperatures. This result is puzzling, because ac conductivity 18 and angle resolved photoemission (ARPES) 19 both show the generally expected increase in scattering at higher frequencies.

For the moment, we assume this behavior may result from other energy scales in the problem not treated by the references above. (The $\pi-\pi$ resonance, superconducting gap energy, and $\omega \sim T$ all occur around $300-500 \mathrm{~cm}^{-1}$.) Accordingly, we will focus on the lowfrequency limit to critically evaluate the Lorentzian theories. We plot the dc limit values $\gamma_{H}^{* d c}=\lim _{\omega \rightarrow 0} \gamma_{H}^{*}(\omega)$ and $\omega_{H}^{* d c}=\lim _{\omega \rightarrow 0} \omega_{H}^{*}(\omega)$ in the inset of Fig. 3 as averaged from 20 to $80 \mathrm{~cm}^{-1}$. At $95 \mathrm{~K}$ the data demonstrate consistency with previous experiments plotted as open squares [13]. Looking at the temperature dependence, we immediately see that $\gamma_{H}^{* d c}(T) \sim T$ and $\omega_{H}^{* d c}(T) \sim 1 / T$. This confirms the Lorentzian behavior proposed in one spinon-holon model [7] and in a skew-scattering model [11. However, this data does not support the other Lorentzian theories which posit a temperature independent numerator and a scattering rate quadratic in temperature $[5,6,6$, 10]. We reemphasize, however, that all of the Lorentzian theories break down at moderate frequecies above $100 \mathrm{~cm}^{-1}$.

As an alternative to the linear-scattering Lorentzian model we consider a square-scattering form for the Hall angle, which corresponds to a Lorentzian-squared ac response. This form was predicted by Varma and Abrahams in their marginal Fermi liquid treatment of Hall scattering in the cuprates 12,20. Extending their result to finite frequencies, we get the square-Lorentzian form:

$$
\theta_{H}(\omega)=\frac{\omega_{H}^{*} \Omega_{p}^{*}}{\left(\Gamma_{H}^{*}-i \omega\right)^{2}}
$$

$\omega_{H}^{*}$ is still the cyclotron frequency, linear in $B$, and $\Omega_{p}^{*}$ is indicative of a new energy scale in the problem, interpreted as a Fermi surface average of the scattering derivative [12]. Solving for the square-scattering rate $\Gamma_{H}^{*}$ and weighting parameter $\omega_{H}^{*} \Omega_{p}^{*}$, one sees at once the independence of $\omega_{H}^{*} \Omega_{p}^{*}$ from both temperature and frequency in Fig. 1 . The existence of such a robust parameter that is constant over 20-250 $\mathrm{cm}^{-1}$ in infrared energy and 95-190 $\mathrm{K}$ in temperature is remarkable, and provides convincing evidence that the square-Lorentzian analysis may elucidate the dominant physics. In the dc limit, the scattering rate, $\Gamma_{H}^{* d c}$, is again linear in temperature, but with twice the value as in the Lorentzian analysis. At higher frequencies $\Gamma_{H}^{*}(\omega)$ now shows a moderate increase, qualitatively consistent with scattering rates observed in ac conductivity [18] and ARPES [19]. 
Although the square-Lorentzian functional form for the Hall angle is a valid causal response function, it cannot be correct at all frequencies since it leads to a Hall sum of zero [16]: $\omega_{H}=\frac{2}{\pi} \int_{0}^{\infty} \operatorname{Re}\left(\tan \left[\theta_{H}(\omega)\right]\right) d \omega=0$, inconsistent with the finite $\omega_{H}$ implied by ARPES measurements of the Fermi surface [19] and band theory. The positive Hall angle measured at $1000 \mathrm{~cm}^{-1}$ by Cerne et al. [15] is an indication that the functional form is already changing. The exact behavior of this crossover is therefore an interesting topic for further investigation.

It is interesting to consider not only $\Gamma_{H}^{*}$, the renormalized ac observable deduced from the square-Lorentzian analysis, but also the bare scattering $\Gamma_{H}$. At dc, the bare and renormalized values satisfy $\omega_{H} \Omega_{p} / \Gamma_{H}{ }^{2}=$ $\omega_{H}^{*} \Omega_{p}^{*} / \Gamma_{H}^{* 2}$. This bare Hall scattering $\Gamma_{H}$ is linear in temperature just as the bare longitudinal scattering $\gamma_{o}$ is linear in temperature as seen in the famous $\rho \sim T$ relation. The renormalizations enter differently, however, between longitudinal and Hall transport since $\Gamma_{H}^{*}$ stays linear in temperature, but $\gamma_{o}^{*}$ increases superlinearly due to a decreasing renormalized transport mass $m_{o}^{*}$ [18]. This difference might be accounted for with vertex corrections in the Kubo formula which enter differently in $\sigma_{x x}$ and $\sigma_{x y}$. The $\cot \left(\theta_{H}\right) \sim \Gamma_{H}^{*}{ }^{2}$ form of the Hall angle obtained by ref. 12] is itself a consequence of vertex corrections in $\sigma_{x y}$. Therefore, the relation between $\gamma_{o}^{*}$ and $\Gamma_{H}^{*}$ presents an interesting subject for future theoretical and experimental work.

In summary, we have measured the complex Hall response at infrared frequencies. The observed $\theta_{H}(\omega)$ does not fit the Lorentzian lineshape predicted by many models of transport in the cuprates or conventional transport theory; only at low frequencies is the data consistent with a subset of linear-scattering models [7],11 that predict the observed behavior: $\gamma_{H}^{*}(T) \sim T, \omega_{H}^{*}(T) \sim 1 / T$. Alternately, the data show a good fit to a square-Lorentzian form, over the entire frequency range, with the temperature dependence of the response function coming again from a relaxation rate linear in temperature, $\Gamma_{H}^{*}(T) \sim T$. In all cases, the experiments suggest that transport in the cuprates is governed throughout by a relaxation rate that is linear in temperature.

Acknowledgement - The authors are grateful to E. Abrahams, J. Cerne, A. Millis, N. P. Ong, C. M. Varma and V. Yakovenko for insightful discussions.

This work was supported in part by the NSF (Grant No. DMR0070959). M.G. thanks the A.v. Humboldt Foundation for support during the writing of this paper.

* Current address: Walter Schottky Institut, Technische Universität München, D-85748 Garching, Germany; email: mgrayson@alumni.princeton.edu

† Current address: Laboratoire National des Champs Magnétiques Pulsés, 143 Avenue de Rangueil, 31432 Toulouse, France
[1] T. R. Chien, D. A. Brawner, Z. Z. Wang and N. P. Ong, Phys. Rev. B 43, 6242 (1991); J. P. Rice, J. Giapintzakis, D. M. Ginsberg and J. M. Mochel, Phys. Rev. B 44, 10158 (1991).

[2] J. M. Harris, Y. F. Yan and N. P. Ong, Phys. Rev. B 46, 14293 (1992); K. Semba and A. Matsuda, Phys. Rev. Lett. 86, 496 (2001).

[3] T. R. Chien, Z. Z. Wang and N. P. Ong, Phys. Rev. Lett. 67, 2088 (1991); Wu Jiang, J. L. Peng, S. J. Hagen and R. L. Greene, Phys. Rev. B 46, 8694 (1992); P. Xiong, G. Xiao and X. D. Wu, Phys. Rev. B 47, 5516 (1993); M. D. Lan, J. Z. Liu, Y. X. Jia, Lu Zhang and R. N. Shelton, Phys. Rev. B 49, 580 (1994); A. Carrington, A. P. Mackenzie, C. T. Lin and J. R. Cooper, Phys. Rev. Lett 69, 2855 (1992).

[4] C. Kendziora, D. Mandrus, L. Mihaly and L. Forro, Phys. Rev. B 46, 14297 (1992); G. Xiao, P. Xiong and M. Z. Cieplak, Phys. Rev. B 46, 8687 (1992).

[5] P. W. Anderson, Phys. Rev. Lett. 67, 2092 (1991).

[6] P. Coleman, A. J. Schofield, A. M. Tsvelik, Phys. Rev. Lett. 76, 1324 (1996).

[7] D. K. K. Lee, P. A. Lee, cond-mat/9610075 (1996).

[8] L. B. Ioffe and A. J. Millis, Phys. Rev. B 58, 11631 (1998); D. van der Marel, Phys. Rev. B 60, R765 (1999);

[9] A. T. Zheleznyak, V. M. Yakovenko, H. D. Drew and I. I. Mazin, Phys. Rev. B 57, 3089 (1998); A. T. Zheleznyak, V. M. Yakovenko, and H. D. Drew, Phys. Rev. B 59, 207 (1999).

[10] B. P. Stojkovic and D. Pines, Phys. Rev. B 55, 8576 (1997).

[11] G. Kotliar, A. Sengupta, and C. M. Varma, Phys. Rev. B 53, 3573 (1996).

[12] C. M. Varma and E. Abrahams, Phys. Rev. Lett. 86, 4652 (2001); Phys. Rev. Lett. 88, 139903 (2002).

[13] S. G. Kaplan, S. Wu, H.-T. S. Lihn and H. D. Drew, Q. Li, D. B. Fenner, J. M. Phillips, S. Y. Hou, Phys. Rev. Lett. 76, 696 (1996).

[14] B. Parks, S. Spielman, J. Orenstein, Phys. Rev. B 56, 115 (1997).

[15] J. Cerne, M. Grayson, D. C. Schmadel, G. S. Jenkins, H. D. Drew, R. Hughes, A. Dabkowski, J. S. Preston and P.-J. Kung, Phys. Rev. Lett. 84, 3418 (2000).

[16] H. D. Drew and P. Coleman, Phys. Rev. Lett. 78, 1572 (1997).

[17] J. W. Allen, and J. C. Mikkelsen, Phys. Rev. B 15, 2952 (1977).

[18] Z. Schlesinger, R. T. Collins, F. Holtzberg, C. Feild, S. H. Blanton, U. Welp, G. W. Crabtree, Y. Fang, and J. Z. Liu, Phys. Rev. 65, 801 (1990); A. V. Puchkov, D. N. Basov, and T. Timusk, J. Phys.: Cond. Matt. 8, 10049 (1996).

[19] T. Valla, et al., Science 285, 2110 (1999); A. Kaminski, et al., Phys. Rev. Lett. 84, 1788 (2000).

[20] R. Hlubina (Phys. Rev. 64, 132508 (2001)) and V. Yakovenko (private communication) have also analyzed this model and have concluded that the square scattering term cannot be greater than the linear-scattering term. Lineshape fits to the data can accomodate an additional linear-scattering component that is as large as the squareLorentzian term so none of the above analyses are clearly 
ruled out by experiment.

FIG. 1. The measured complex Faraday angle. Insets shows separate experimental setups for measuring $\operatorname{Re}\left(\theta_{F}\right)$ and $\operatorname{Im}\left(\theta_{F}\right)$. The Kramers-Kronig transform of $\operatorname{Re}\left(\theta_{F}\right)$ is plotted in gray.

FIG. 2. The derived complex Hall angle. Inset shows transmission data with extended Drude conductivity fits for each temperature in dashed lines.

FIG. 3. Lorentzian parameterization of Hall angle. The Hall scattering $\gamma_{H}$ develops strong downwards frequency dependence above $100 \mathrm{~cm}^{-1}$, implying a deviation from simple Lorentzian behavior. Inset plots the low-frequency limit of these parameters vs. T.

FIG. 4. Square-Lorentzian parameterization of Hall angle. The scattering parameter $\Gamma_{H}$ shows a moderate increase with frequency and $\omega_{H} \Omega_{p}$ is notably temperature and frequency independent. Inset plots the low-frequency limit of these parameters vs. T. 


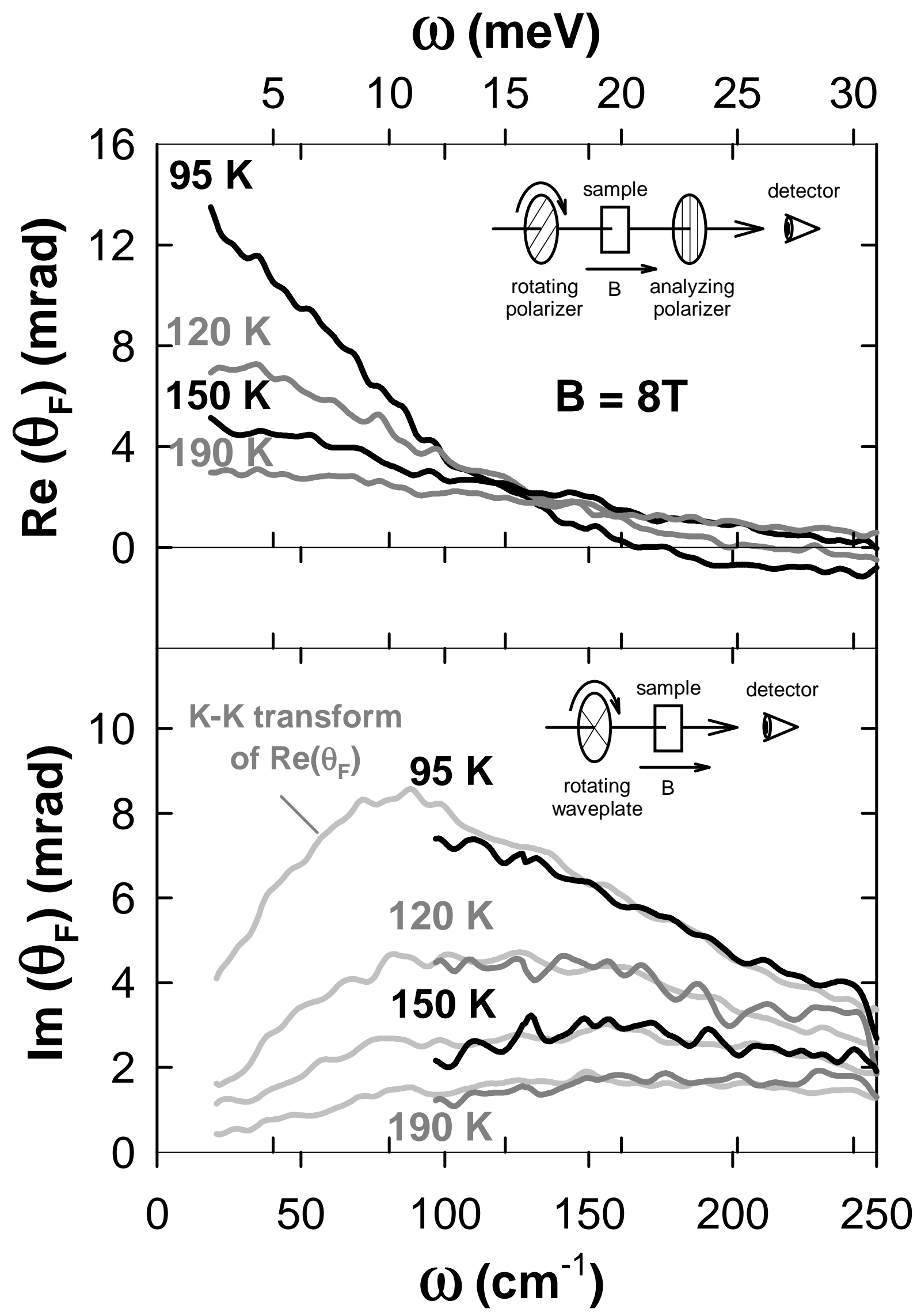




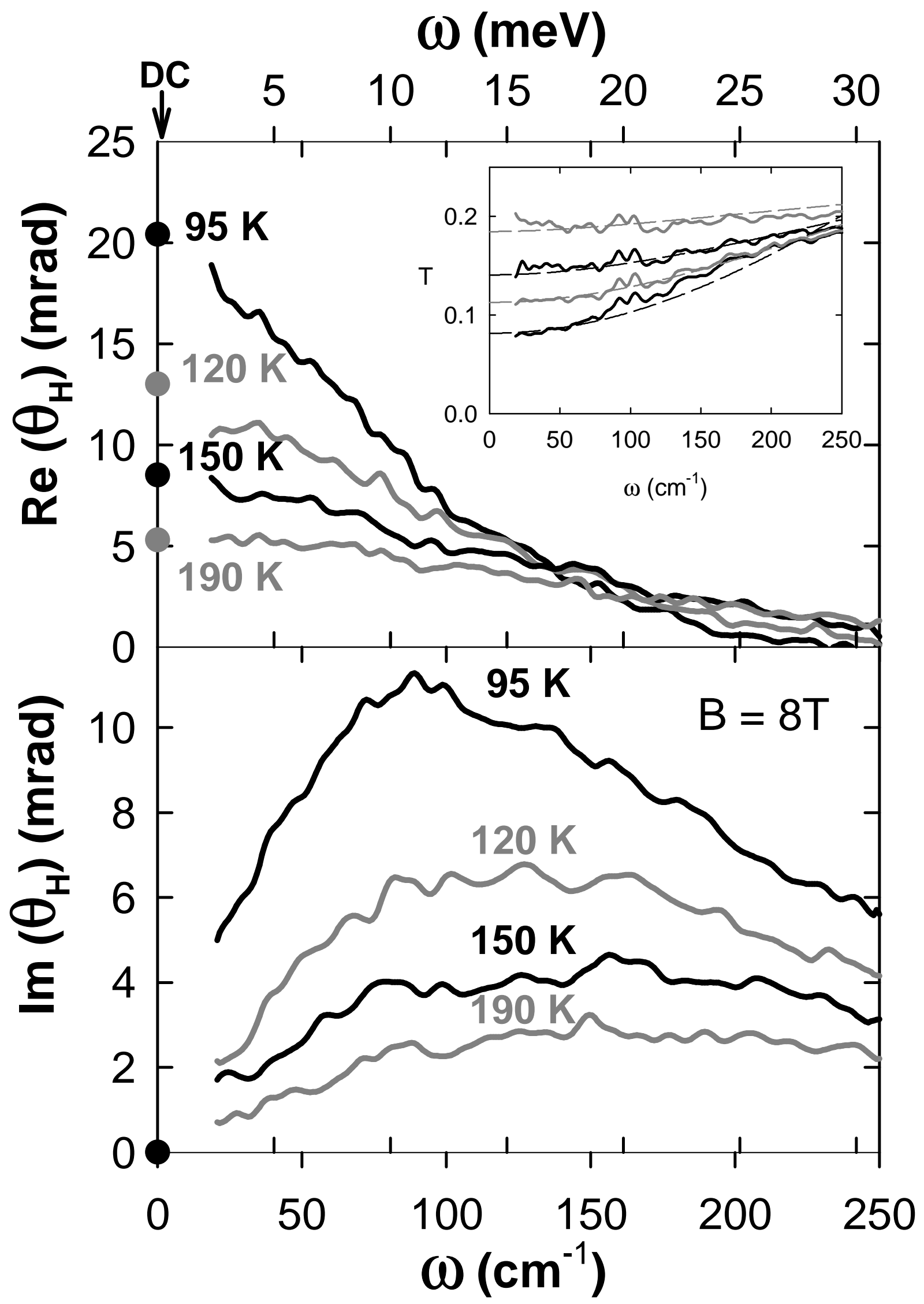




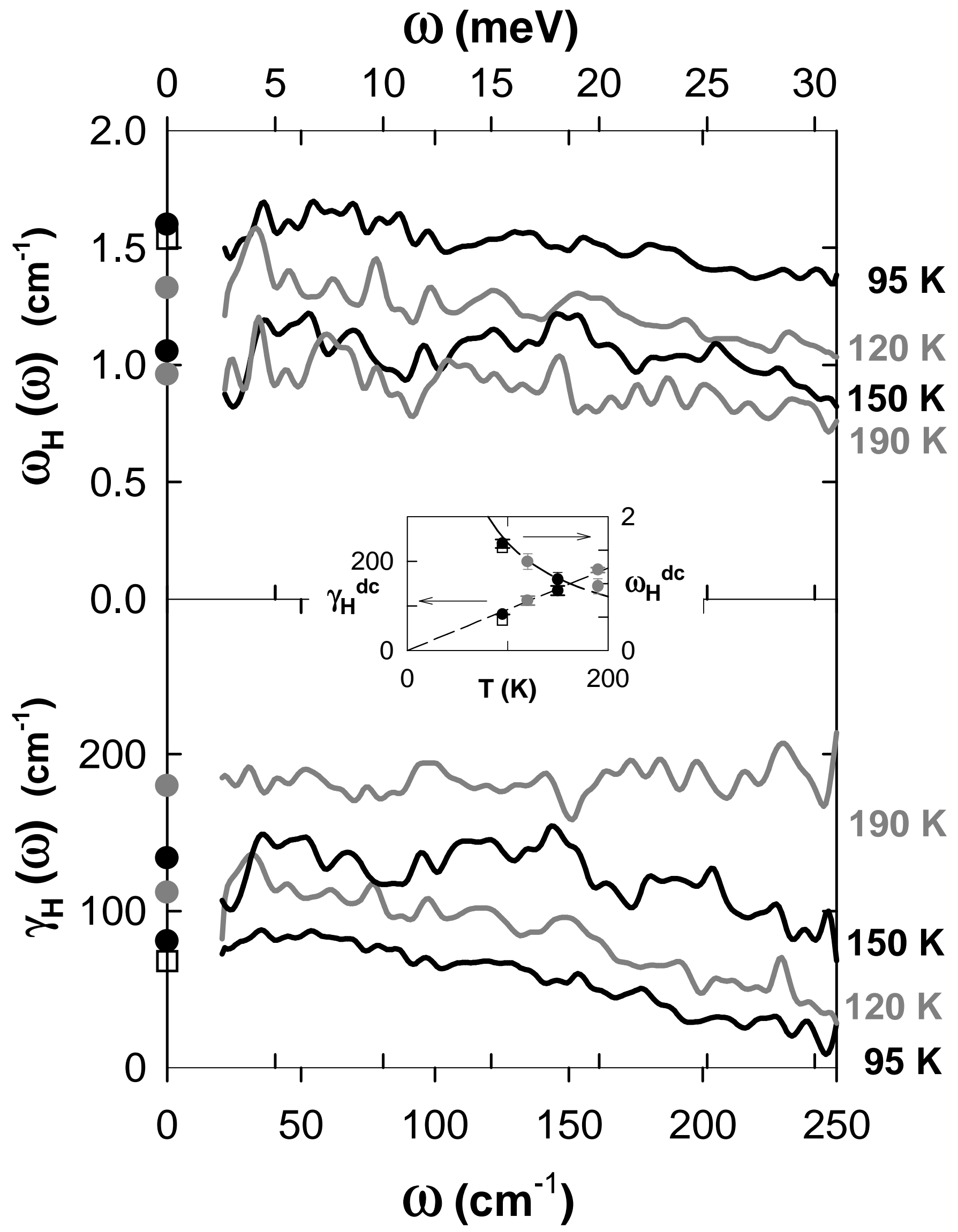




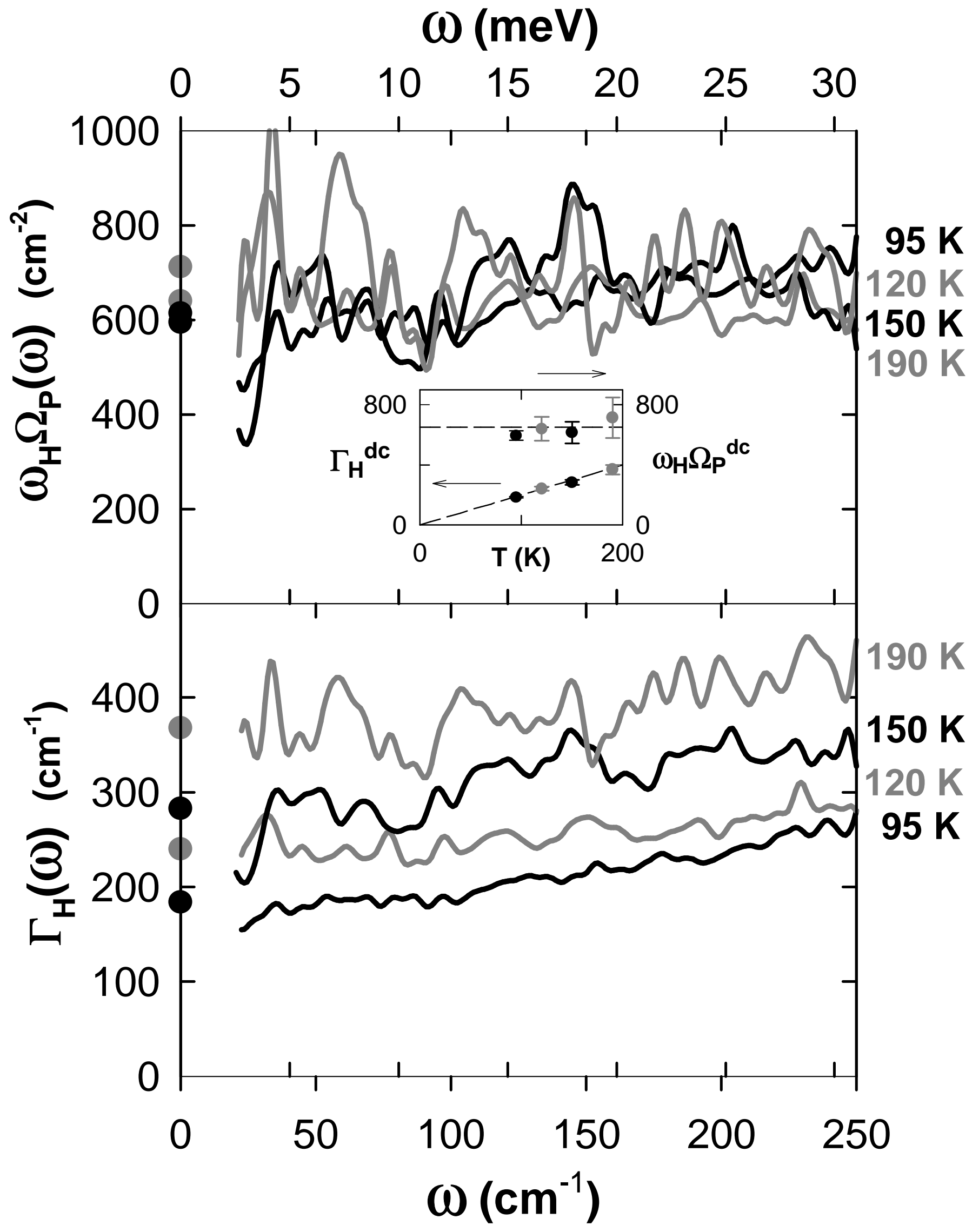

